\title{
Mutational Mapping of RAS-Responsive Domains of the Saccharomyces cerevisiae Adenylyl Cyclase
}

\author{
JOHN COLICELLI, JEFFREY FIELD, ROYMARIE BALLESTER, NICHOLAS CHESTER, \\ DALLAN YOUNG, AND MICHAEL WIGLER* \\ Cold Spring Harbor Laboratory, P.O. Box 100, Cold Spring Harbor, New York, 11724
}

Received 22 December 1989/Accepted 26 February 1990

\begin{abstract}
Large deletion and small insertion mutations in the adenylyl cyclase gene of Saccharomyces cerevisiae were used to map regions required for activation by RAS protein in vitro. The amino-terminal 605 amino acids were found to be dispensable for responsiveness to RAS protein. All other deletions in adenylyl cyclase destroyed its ability to respond to RAS. Small insertion mutations within the leucine-rich repeat region also prevented RAS responsiveness, while other insertions did not.
\end{abstract}

In the yeast Saccharomyces cerevisiae there are two genes, RAS1 and RAS2, that encode GTP-binding proteins with both structural and functional homology to the mammalian RAS proteins $(2,3,10,15)$. In $S$. cerevisiae, these genes are known to play a critical role in determining cellular responses to nutrient conditions $(10,21)$. Although the function of RAS proteins in mammalian cells remains enigmatic, $S$. cerevisiae RAS proteins stimulate adenylyl cyclase (the $C Y R I$ gene product), resulting in elevated levels of the intracellular second messenger cyclic (cAMP). Increased levels of cAMP are able to derepress the cAMP-dependent protein kinases. While a $R A S$ deficiency is normally a lethal event, lethality can be overcome by mutations which increase the constitutive level of cAMP production $(4,9)$, decrease cAMP hydrolysis (14), or uncouple the protein kinases from cAMP regulation $(18,21)$.

We have been particularly interested in elucidating the mechanism of RAS-effector interactions in general and adenylyl cyclase stimulation in particular. We have previously described the purification of yeast adenylyl cyclase and an in vitro RAS activation assay using yeast RAS2 protein purified from bacteria (6). We describe here the use of this assay to analyze mutants of adenylyl cyclase in an effort to determine the regions required for RAS interaction and stimulation.

\section{MATERIALS AND METHODS}

Strains, media, and transformations. Plasmids were propagated in Escherichia coli HB101. Bacterial transformations were performed by using a standard procedure. The yeast strain SPK-3T3 (MATa his3 leu2 ura3 trpl ade8 canl ras $1:: H I S 3$ ras2::URA3 pTPK3-TRP1) is a plasmid exchange derivative of SPK-3 (20). Yeast cultures were grown in either rich medium (YPD) or synthetic medium (SC) with appropriate auxotrophic supplements. Yeast transformations were performed by using lithium acetate (8). SPK-3T3 cells were transformed with pYCYR or with mutant constructs which contain the LEU2 marker. Transformants were grown in YPD for 2 days in order to allow segregation of the pTPK3-

\footnotetext{
* Corresponding author.
}

TRP1 plasmid. Segregation analysis yielded cells that were $\mathrm{Leu}^{+} \mathrm{Trp}^{-}$and that were fully dependent on the extrachromosomal adenylyl cyclase plasmid for viability.

Plasmids and DNA manipulations. The construction of the plasmid pYCYR has been described previously (7). Deletion la was produced by digestion of pYCYR with KpnI and MluI followed by treatment with mung bean exonuclease to produce blunt ends and with calf intestinal phosphatase. The resulting linear DNA was ligated in the presence of a NotI linker oligonucleotide. The resulting junction was sequenced $(1,17)$ to verify that the reading frame was not altered. Deletion 3a was created by cleaving pEF-CYR1 (6) with $S a c I$ followed by religation at a dilute concentration, resulting in the loss of a 3.6-kilobase-pair SacI fragment. The 4.7-kilobase SalI-to-NcoI fragment of this deletion mutant was then ligated to the 8.8-kilobase SalI-to-NcoI fragment of pYCYR. All other deletions were constructed by using the polymerase chain reaction (PCR). This was accomplished by the utilization of primers that introduce new restriction endonuclease recognition sites. The restriction site chosen is positioned to be in frame with, but displaced from, an identical restriction site in adenylyl cyclase. Deletions were designed such that they are not accompanied by the addition of any amino acid residues, except for group III (see below). Exchange of a wild-type fragment with the PCR product resulted in the deletion of sequences between the newly introduced and preexisting restriction sites. Standard PCR conditions (16) were used, and reactions were stopped after 15 cycles. Each primer had 18 bases of perfect complimentation to adenylyl cyclase sequences. Primers that introduce new restriction sites included the recognition site sequence plus either two or four additional nucleotides at their $5^{\prime}$ ends. Group I deletions (exclusive of $1 \mathrm{a}$ and 3a) were made by introducing a new SalI site and deleting sequences up to the preexisting SalI site at nucleotide position 656. Similarly, group II deletions were made by introducing new NcoI sites upstream of the endogenous $\mathrm{NcoI}$ site at nucleotide position 5485. Group III deletions were created by adding back PCR-generated fragments into the NcoI site of $\Delta 5$. The resulting large deletions had an additional $\mathrm{Ncol}$ site and a 2-amino-acid insertion. In the case of IN6, a full sequence replacement was made, with the net result of a 3-amino-acid insertion. Group IV deletions were made by introducing a 
new $X$ hoI site $(\Delta 11$ and $\Delta 13)$ or a $S a c I$ site $(\Delta 12$ and $\Delta 14)$. The endogenous $X h o I$ site and $S a c I$ site are at nucleotide positions 3858 and 4085 , respectively. Insertion mutations were created by standard oligonucleotide-directed mutagenesis $(12,25)$. The oligonucleotides used contained the insert sequence GGATCCGGA flanked by 15 nucleotides of perfect complementation on each side. The insert sequence encodes Gly-Ser-Gly and contains a BamHI endonuclease recognition site. Insertions were confirmed by BamHI digestions.

Adenylyl cyclase purification and analysis. Yeast strains harboring adenylyl cyclase constructs were grown to late log phase in 1 liter of YPD. Adenylyl cyclase was purified by immunoprecipitation from membrane preparations obtained from 1-liter cultures (6). Catalytic activity determinations were performed as previously described (6). RAS2 ${ }^{\text {val19 }}$ proteins was used in these assays to stimulate cyclase activity. We have previously shown that wild-type RAS2 protein will also work (5). Although the strains used do contain wild-type adenylyl cyclase, it does not appear to copurify with the epitope-tagged adenylyl cyclases (unpublished results) and does not contribute to the measured adenylyl cyclase activities. Immunoaffinity-purified adenylyl cyclase was subjected to sodium dodecyl sulfate-polyacrylamide gel electrophoresis, transferred electrophoretically to nitrocellulose, and probed with the monoclonal antibody $12 \mathrm{CA5}(6)$ raised against the peptide epitope. Antibody $12 \mathrm{CA} 5$ was used at a concentration of $3 \mu \mathrm{g} / \mathrm{ml}$. The immunoblot was then developed with colloidal gold conjugated to goat anti-mouse immunoglobulin $\mathrm{G}$ followed by gold enhancement (Bio-Rad Laboratories). Molecular weight markers were obtained from Sigma Chemical Co.

\section{RESULTS}

Central to our studies is a yeast adenylyl cyclase expression system that expresses high levels of the enzyme. The adenylyl cyclase expression construct, pYCYR, uses the strong yeast $\mathrm{ADH} 1$ promoter to express adenylyl cyclase fused to a short amino-terminal epitope (Fig. 1). This epitope is used for immunopurification. The expression plasmid also contains origins of replication and selectable markers for growth in yeast and bacteria. Its construction has been described previously (7). Two other features of adenylyl cyclase are highlighted in Fig. 1. The carboxy-terminal 418 amino acids, designated as the catalytic domain, are sufficient for $\mathrm{Mn}^{2+}$-dependent cyclase activity but are insufficient for activation by RAS protein and GTP in the presence of $\mathrm{Mg}^{2+}(9)$. The region from amino acid 733 to 1301 , which we call the leucine-rich repeat region, is composed of 26 copies of an approximately 23 -amino-acid repeating unit that has a high content of aliphatic residues, especially leucine. The consensus repeat unit is represented in Fig. 1. Mutants in adenylyl cyclase were generated and tested as described below.

Plasmids carrying the mutant adenylyl cyclase gene were transformed into the yeast strain SPK-3T3, which has both $R A S$ genes disrupted. Since $R A S$ is normally essential for cell viability, this strain is kept alive with an extrachromosomal plasmid, pTPK3-TRP1, that overexpresses $T P K 3$, a gene encoding a catalytic component of the cAMP-dependent protein kinase (19). All of our adenylyl cyclase constructs expressed the catalytic portion of the enzyme, and all were able to suppress the lethality of a $R A S$ deficiency in this strain, as determined by plasmid exchange (see Materials

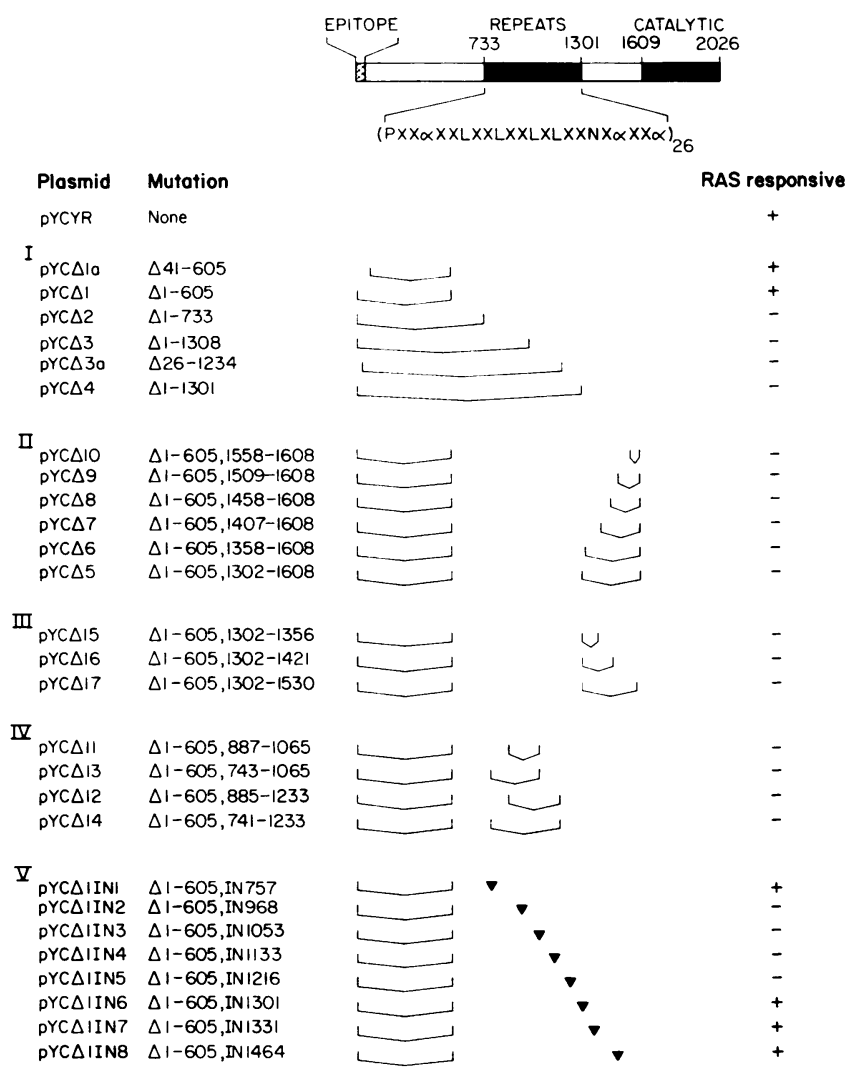

FIG. 1. Mutational analysis of adenylyl cyclase. A linear representation of the adenylyl cyclase protein with the amino-terminal peptide epitope is shown. The leucine-rich repeat region and the catalytic domain are also labeled, and the amino acid positions which demarcate their boundaries are given. The consensus repeat unit is shown $(P$, proline; $L$, leucine; $N$, asparagine; $X$, any amino acid residue; and $\alpha$, any aliphatic amino acid from the group methionine, leucine, isoleucine, and valine). The leftmost column gives the name of each mutant; this is followed by the positions of the amino acid residues (inclusive) which have been deleted $(\Delta)$ and the position of the amino acid residue immediately before an insertion (IN). Deletions are shown graphically with brackets, and insertions are indicated with arrowheads. Mutants were scored for RAS responsiveness by using adenylyl cyclase assays as described in the text. For each deletion mutant and IN6, which were prepared by PCR, cell extracts were prepared from two independent constructs. Testing of independent PCR constructs controlled for the possibility that additional, unforeseen mutations were introduced in the PCR and cloning process. A single construct of each of the other insertion mutants was analyzed. At least two independent adenylyl cyclase activity measurements were performed on each extract to determine RAS responsiveness.

and Methods). We were thus able to obtain yeast strains that have lost the pTPK3-TRPI plasmid but contain the mutated $C Y R I$ gene. [These strains show no phenotypic abnormalities associated with excessive CYR1 expression (i.e., heat shock sensitivity).] Adenylyl cyclase was prepared from these strains by immunoaffinity purification (6), and activity assays were performed in the presence of $\mathbf{M n}^{2+}, \mathbf{M g}^{2+}$, or $\mathrm{Mg}^{2+}$ and RAS2 protein. Typical results are shown in Table 1. All mutants had appreciable levels of adenylyl cyclase activity with $\mathrm{Mn}^{2+}$, indicating that the catalytic function was not substantially altered and that no frameshift mutations had been introduced. Some variability was observed in the 
TABLE 1. Activation of mutant adenylyl cyclase protein by RAS2 ${ }^{a}$

\begin{tabular}{lcccc}
\hline \multirow{2}{*}{ Strain } & \multicolumn{3}{c}{$\begin{array}{c}\text { Adenylycyclase activity (pmol of } \\
\text { cAMP generated/30 min) with: }\end{array}$} & $\begin{array}{c}\text { Fold stimulation } \\
\text { by } \mathrm{RAS}^{b}\end{array}$ \\
\cline { 2 - 4 } & $\mathrm{Mn}^{2+}$ & $\mathbf{M g}^{2+}$ & $\mathrm{Mg}^{2+}+\mathrm{RAS2}$ & \\
\hline Wild type & 165 & 10.8 & 204 & $19.0^{c}$ \\
$\Delta 1 \mathrm{a}$ & 334 & 33.5 & 195 & 5.8 \\
$\Delta 1$ & 501 & 57.8 & 495 & 8.6 \\
$\Delta 2$ & 306 & 27.7 & 46.4 & $1.7^{d}$ \\
$\Delta 3$ & 459 & 50.8 & 33.5 & 0.6 \\
$\Delta 4$ & 359 & 36.7 & 30.9 & 0.8 \\
\hline
\end{tabular}

${ }^{a}$ Adenylyl cyclase was immunopurified from yeast membrane preparations as described previously and assayed in the presence of $\mathrm{Mn}^{2+}, \mathrm{Mg}^{2+}$, or $\mathrm{Mg}^{2+}$ plus RAS2 $2^{\text {val19 }}$ protein bound to GTP (6). The amount of each extract tested was chosen to give roughly comparable levels of activity in the presence of $\mathrm{Mn}^{2+}$ (approximately $10 \mu \mathrm{g}$ of protein as crude extract before immobilization with antibody). Each value presented is the average of two measurements.

${ }^{b}$ The fold stimulation due to RAS is calculated as (activity measured with $\mathrm{Mg}^{2+}$ plus RAS)/(activity measured with $\mathbf{M g}^{2+}$ ).

$c$ The observed RAS-dependent stimulation of wild-type adenylyl cyclase was usually comparable with that of $\Delta 1 \mathrm{a}, \Delta 1$, and the other RAS-responsive insertion mutants. It has, however, been measured at lower or higher levels (as presented here).

${ }^{d}$ The slight stimulation was not seen when the assay was repeated.

absolute values of adenylyl cyclase activity within these assays, due in part to differential yields from various extracts. Mutants were classified as RAS responsive if activity levels with $\mathrm{Mg}^{2+}$ plus RAS were at least fourfold greater than levels of activity measured with $\mathrm{Mg}^{2+}$ alone. Mutants were classified as unresponsive if activity levels with $\mathbf{M g}^{2+}$ plus RAS were less than twofold greater than levels of activity measured with $\mathbf{M g}^{2+}$ alone. All mutants could be clearly classified. In addition, for RAS-responsive mutants and wild-type strains, adenylyl cyclase activity in the presence of $\mathrm{Mg}^{2+}$ and RAS was consistently at least $50 \%$ of $\mathrm{Mn}^{2+}$ activity. For nonresponsive mutants, this activity was only $15 \%$ or less of the corresponding $\mathrm{Mn}^{2+}$ activity.

The first set of deletion mutations we constructed began at or near the amino terminus of adenylyl cyclase and had sequences up to and including the leucine-rich repeat region removed (Fig. 1, group I). Because the epitope-encoding sequences are not affected by the mutagenesis, all mutants possesed the same 20 amino acids at their amino termini. Adenylyl cyclases derived from these mutants were analyzed by sodium dodecyl sulfate-polyacrylamide gel electrophoresis and were found to have mobilities corresponding to their predicted sizes (Fig. 2). Two deletions of $C Y R I, \Delta 1$ a and $\Delta 3 \mathrm{a}$, were made by restriction endonuclease digestion and religation. Other deletion mutations in the adenylyl cyclase-coding sequences were created by using a more complicated strategy (described in Materials and Methods). Fragments of the $C Y R 1$ gene were synthesized in a PCR, and these fragments were used to construct deletion mutants. While all of the mutants retained high levels of $\mathrm{Mn}^{2+}$. dependent activity, only deletions $\Delta 1 \mathrm{a}$ and $\Delta 1$ remained responsive to $\mathrm{RAS}$ in the presence of $\mathrm{Mg}^{2+}$. Thus, the $\mathrm{N}$-terminal 605 amino acids, up to 127 amino acids before the start of the leucine-rich repeat region, are dispensable.

To determine the importance of the leucine-rich repeat region and the sequences lying between the repeats and the catalytic domain, further deletions were created, beginning with $\Delta 1$, the smallest responsive construct, as the parent (Fig. 1). The group II deletions all began at the start of the catalytic domain and removed part or all of the sequences up to the leucine rich repeat region. Group III deletions are

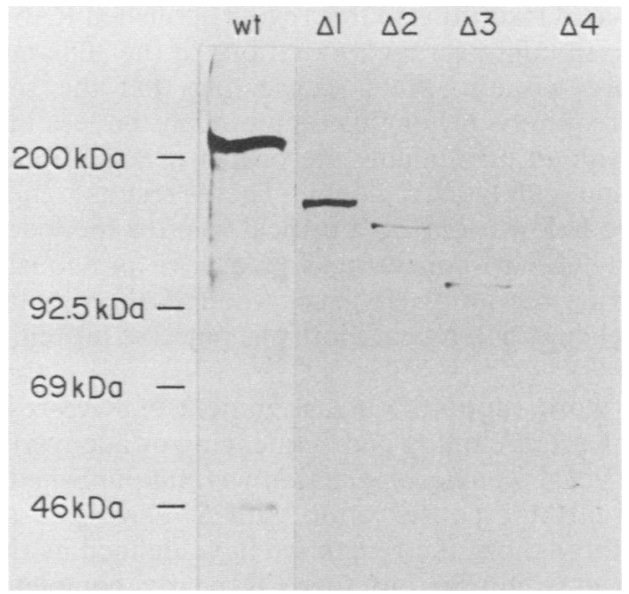

FIG. 2. Western (immuno-) blot of deleted adenylyl cyclases. Immunopurified adenylyl cyclases (wild type [wt] and mutant) were run on a sodium dodecyl sulfate-polyacrylamide gel, transferred to nitrocellulose, and probed with a monoclonal antibody against the peptide epitope (see Materials and Methods). Each lane is labeled to indicate the source of adenylyl cyclase (wild type or deletion mutant), and the positions of molecular mass markers are indicated on the left. The blot is a composite of two experiments run separately. kDa, Kilodaltons.

located in the same area but initiate from the end of the leucine-rich repeat region and proceed toward the catalytic domain. None of the deletion mutants in this region retained RAS responsiveness. Group IV deletions were confined to the leucine-rich repeat region and were designed so that an integral number of repeat units was removed in each case. Deletion endpoints were at equivalent positions within each repeat. These mutants were also found to be unresponsive to RAS.

Finally, a series of insertion mutations (group V) was made within the repeat region and the sequences preceding the catalytic domain. All insertions were 3 amino acids in length, and their positions are indicated in Fig. 1. IN1-5, -7, and $\mathbf{- 8}$ were made by standard oligonucleotide-directed mutagenesis (see Materials and Methods) and introduced the amino acids Gly-Ser-Gly. IN6 was created by using PCR (see Materials and Methods) and contained the extra amino acids Ala-Met-Ala. While all of the insertions are located within areas determined to be required by analysis of the deletion mutants, only IN2, $-3,-4$, and -5 were found to result in a loss of RAS responsiveness. These inserts map within the leucine-rich repeat region, and each resides at a nonequivalent position within the repeat unit.

\section{DISCUSSION}

Our results demonstrate that the amino-terminal 605 amino acids of adenylyl cyclase are dispensable for RAS responsiveness. The smallest responsive construct, $\Delta 1$, includes, in addition to the catalytic domain, the 569-aminoacid leucine-rich repeat region and a 308-amino-acid region between the repeat and catalytic domains. Deletion mutations in either of these domains destroyed RAS responsiveness. In addition, the 127 amino acids immediately upstream of the leucine-rich repeat region appear to be required for full RAS responsiveness. The structural constraints in the leucine-rich repeat appear to be quite exacting. Even small, 
3-amino-acid insertions in this region abolished RAS responsiveness. In contrast, such mutations in the 308-amino-acid region are without effect (suggesting that the sequencespecific requirement for this region might be less important than its role in maintaining the proper spacing between the repeat and catalytic regions). These results, and others described below, point to a critical role for the leucine-rich repeat in RAS responsiveness. We have previously noted the growing literature on gene products that contain very similar leucine-rich repeat motifs as putative protein-binding domains (7).

Other work supports our assignment of RAS-responsive regions. Recent cloning and sequencing of adenylyl cyclase from the yeast Saccharomyces kluyveri (unpublished results) revealed striking conservation with $S$. cerevisiae adenylyl cyclase throughout the region we have defined as the RASresponsive structure, but there is nearly complete divergence amino terminal to this region. Further studies are required to determine whether this pattern of sequence conservation reflects a functional conservation with regard to RAS stimulation. The requirement of the leucine-rich repeat region is also supported by experimentation based upon an in vivo interference assay. Some adenylyl cyclase mutants can dominantly interfere with the expression of the heat shock phenotype resulting from a $R A S 2^{\text {val19 }}$ mutation. Interference can be achieved by expression of little more than the leucine-rich repeat region, and mutations within this area abolish interference (7). The requirement for the 308amino-acid region between the leucine-rich repeat and the catalytic domain is supported by observations made by Marshall et al. (13). They developed a series of attenuated $R A S$ mutants that were impaired in their ability to stimulate adenylyl cyclase. These $R A S$ mutants were used to isolate and characterize a compensator mutation within adenylyl cyclase that was located at position 1547 . Our work is in conflict with the findings of Uno et al., who claim that little more than the catalytic domain of adenylyl cyclase is sufficient for RAS responsiveness (22).

In addition to the regions already discussed, important RAS-responsive elements may lie within the catalytic domain. The extent of amino acid residues actually devoted to the catalysis of cAMP formation may be quite modest as judged by the level of sequence conservation among adenylyl cyclases of different species $(11,23,24)$. The two functions, catalysis and RAS responsiveness, may be intimately associated and difficult to analyze separately. Some data implicating catalytic domain sequences in RAS responsiveness have recently been reported (23). Finally, we wish to emphasize that nothing in our studies requires that adenylyl cyclase interact directly with RAS. The adenylyl cyclase obtained from yeast purifies as a complex with at least one other protein component, a 70-kilodalton subunit. The role of this protein is currently under study. The loss of RAS responsiveness in a mutant adenylyl cyclase might reflect an altered interaction with other elements of the adenylyl cyclase complex.

\section{ACKNOWLEDGMENTS}

We thank P. Bird for preparation of the manuscript.

This work was supported by Public Health Service grants from the National Institutes of Health (NIH), by grants from the American Cancer Society, and by the Pfizer Biomedical Research Award. J.F. and R.B. are supported by NIH fellowships. M.W. is an American Cancer Society Research Professor.

\section{LITERATURE CITED}

1. Biggin, M. D., T. J. Gibson, and G. F. Hong. 1983. Buffer gradient gels and $35 \mathrm{~S}$ label as an aid to rapid DNA sequence determination. Proc. Natl. Acad. Sci. USA 80:3963-3965.

2. DeFeo-Jones, D., E. M. Scolnick, R. Koller, and R. Dhar. 1983. ras-related gene sequences identified and isolated from Saccharomyces cerevisiae. Nature (London) 306:707-709.

3. DeFeo-Jones, D., K. Tatchell, L. C. Robinson, I. Sigal, W. Vass, D. R. Lowy, and E. M. Scolnick. 1985. Mammalian and yeast ras gene products: biological function in their heterologous systems. Science 228:179-184.

4. De Vendittis, E., A. Vitelli, R. Zahn, and O. Fasano. 1986. Suppression of defective RAS1 and RAS2 functions in yeast by an adenylate cyclase activated by a single amino acid change. EMBO J. 5:3657-3663.

5. Field, J., D. Broek, T. Kataoka, and M. Wigler. 1987. Guanine nucleotide activation of, and competition between, RAS proteins from Saccharomyces cerevisiae. Mol. Cell. Biol. 7:21282133.

6. Field, J., J. Nikawa, D. Broek, B. MacDonald, R. Rodgers, I. A. Wilson, R. A. Lerner, and M. Wigler. 1988. Purification of a $R A S$-responsive adenylyl cyclase complex from Saccharomyces cerevisiae by use of an epitope addition method. Mol. Cell. Biol. 8:2159-2165.

7. Field, J., H.-P. Xu, T. Michaeli, M. Sass, M. Wigler, and J. Colicelli. 1990. The leucine rich repeat region of the $S$. cerevisiae adenylyl cyclase gene interferes with Ras function. Science 247:464-467.

8. Ito, H., Y. Fukuda, K. Murata, and A. Kimura. 1983. Transformation of intact yeast cells treated with alkali cations. J. Bacteriol. 153:163-168.

9. Kataoka, T., D. Broek, and M. Wigler. 1985. DNA sequence and characterization of the $\mathrm{S}$. cerevisiae gene encoding adenylate cyclase. Cell 43:493-505.

10. Kataoka, T., S. Powers, S. Cameron, O. Fasano, M. Goldfarb, J. Broach, and M. Wigler. 1985. Functional homology of mammalian and yeast $R A S$ genes. Cell 40:19-26.

11. Krupinski, J., F. Coussen, H. A. Bakalyar, W.-J. Tang, G. Feinstein, K. Orth, C. Slaughter, R. R. Reed, and A. G. Gilman. 1989. Adenylyl cyclase amino acid sequence: possible channelor transporter-like structure. Science 244:1558-244.

12. Kunkel, T. 1985. Rapid and effective site-specific mutagenesis without phenotypic selection. Proc. Natl. Acad. Sci. USA 82:488-492.

13. Marshall, M. S., J. B. Gibbs, E. M. Scolnick, and I. S. Sigal. 1988. An adenylate cyclase from Saccharomyces cerevisiae that is stimulated by RAS proteins with effector mutations. Mol. Cell. Biol. 8:52-61.

14. Nikawa, J., S. Cameron, T. Toda, K. M. Ferguson, and $M$. Wigler. 1987. Rigorous feedback control of cAMP levels in Saccharomyces cerevisiae. Genes Dev. 1:931-937.

15. Powers, S., T. Kataoka, O. Fasano, M. Goldfarb, J. Strathern, J. Broach, and M. Wigler. 1984. Genes in S. cerevisiae encoding proteins with domains homologous to the mammalian ras proteins. Cell 36:607-612.

16. Saiki, R., D. Gelfand, S. Stoffe, S. Scharf, R. Higushi, G. Horn, K. Mullis, and H. Erlich. 1988. Primer-directed enzymatic amplification of DNA with a thermostable DNA polymerase. Science 239:489-491.

17. Sanger, F., S. Nicklen, and A. R. Coulson. 1977. DNA sequencing with chain-terminating inhibitors. Proc. Natl. Acad. Sci. USA 74:5463-5467.

18. Toda, T., S. Cameron, P. Sass, M. Zoller, J. D. Scott, B. McMullen, M. Hurwitz, E. G. Krebs, and M. Wigler. 1987. Cloning and characterization of $B C Y 1$, a locus encoding a regulatory subunit of the cAMP-dependent protein kinase in yeast. Mol. Cell. Biol. 7:1371-1377.

19. Toda, T., S. Cameron, P. Sass, and M. Wigler. 1987. Three different genes in the yeast Saccharomyces cerevisiae encode the catalytic subunits of the cAMP dependent protein kinase. Cell 50:277-287.

20. Toda, T., S. Cameron, P. Sass, and M. Wigler. 1988. SCH9, a 
gene of $S$. cerevisiae that encodes a protein distinct from, but functionally and structurally related to, cAMP dependent protein kinase catalytic subunits. Genes Dev. 2:517-527.

21. Toda, T., I. Uno, T. Ishikawa, S. Powers, T. Kataoka, D. Broek, S. Cameron, J. Broach, K. Matsumoto, and M. Wigler. 1985. In yeast, RAS proteins are controlling elements of adenylate cyclase. Cell 40:27-36.

22. Uno, I., U. H. Mitsuzawa, K. Tanaka, T. Oshima, and T. Ishikawa. 1987. Identification of the domain of Saccharomyces cerevisiae adenylate cyclase associated with the regulatory function of RAS products. Mol. Gen. Genet. 210:187-194.
23. Yamawaki-Kataoka, Y., T. Tamaoki, H.-R. Choe, H. Tanaka, and T. Kataoka. 1989. Adenylate cyclase in yeast: a comparison of the genes from Schizosaccharomyces pombe and Saccharomyces cerevisiae. Proc. Natl. Acad. Sci. USA 86: 5693-5697.

24. Young, D., M. Riggs, J. Field, A. Vojtek, D. Broek, and M. Wigler. 1989. The adenylyl cyclase gene from Schizosaccharomyces pombe. Proc. Natl. Acad. Sci. USA 86:7989-7993.

25. Zoller, M., and M. Smith. 1984. Oligonucleotide directed mutagenesis: a simple method using two oligonucleotide primers and a single stranded DNA template. DNA 3:479-488. 